\title{
Arbor
}

\section{Santiago de Compostela, conservación y transformación}

\author{
Xerardo Estévez Fernández
}

Arbor CLXX, 671-672 (Noviembre-Diciembre 2001), 473-488 pp.

Puede afirmarse que en Santiago de Compostela se ha conseguido transformar una ciudad histórica, en los términos que más adelante se explicarán, mediante el planeamiento y la arquitectura. El comienzo, como casi siempre, no resultó fácil, pero el balance es claramente positivo y ha alcanzado un reconocimiento general. Las conclusiones - siempre provisionales- que se deducen de quince años de labor indican que la cuestión esencial es el proyecto: desde la idea hasta el plan hay que recorrer un camino largo y complicado, pero el trayecto entre el plan y su ejecución no es menos complejo, y la mejor forma de abordarlo con garantías es el ejercicio del consenso y el diálogo entre la administración y los ciudadanos.

La ordenación del espacio y de las actividades, la cualificación de las zonas de protección o de construcción, los equipamientos, las infraestructuras, los sistemas generales, la conservación y rehabilitación de edificios y zonas históricas es la base de una economía urbana saneada. La función del planeamiento es diseñar la ciudad y, además, aprovechar sus recursos: no todas sus partes tienen crecer de la misma manera ni con los mismos usos, algunas deben ser protegidas, porque el desarrollo ha de partir de criterios de sostenibilidad. Por eso es importante el ejercicio de la solidaridad urbana basada en la calidad de vida mediante el reparto de la riqueza, apoyado en una política fiscal que distribuya los recursos para implantar servicios, equipamientos e infraestructuras en las áreas más desfavorecidas.

Partiendo de estos presupuestos, compete a la administración local impulsar el proceso de crecimiento económico, combinando adecuadamente lo público con lo privado. La administración tiene que participar 
con los demás agentes en el mercado del suelo y la vivienda, de forma sostenida a lo largo de todo el proceso de planificación y gestión, negociando los acuerdos que garanticen la viabilidad del plan y el mantenimiento de la calidad de los espacios públicos.

Estas son, brevemente enunciadas, algunas de las bases del proceso de transformación que se llevó a cabo en Santiago de Compostela, una ciudad que con su historia y su tradición milenaria, es una referencia indiscutible en el orden de lo que se ha dado en llamar patrimonio de la humanidad.

En los diecisiete años transcurridos entre el año jubilar de 1982 y el pasado de 1999, la producción editorial acerca de Compostela y del Camino de Santiago alcanzó una dimensión sin precedentes, sin perjuicio de la notable literatura producida en todas las lenguas de Europa desde que en el siglo XII Aymerico Picaud diera a conocer su Guía del peregrino, incluida en el Códice Calixtino. Esto nos exime de hacer una introducción extensa al tema. Sin embargo, algunos datos pueden ser útiles para establecer la evolución urbana, un tanto encubierta por la dimensión mediática del fenómeno jacobeo, distorsionado en cierta medida en el contexto actual.

Por razones que no hace al caso dilucidar aquí, cuando se habla de Santiago de Compostela se suele prescindir de su historia antigua. Es ya una convención arrancar del año 813, cuando según la tradición se produce la milagrosa revelación (inventio) del edículo romano que alberga unos restos que son atribuidos al Apóstol Santiago. Los intereses políticos y religiosos del momento, concertados frente a la invasión islámica, hacen de este hallazgo el punto de partida de una amplia operación de reactivación de la vida urbana en el norte de la Península y más allá: se construyen calzadas, puentes, iglesias, hospitales; se crean cofradías y cuerpos de asistencia y defensa y se conceden indulgencias. Así, Compostela se consolida como el tercer gran centro de peregrinación de la cristiandad, junto con Jerusalén y Roma. En el siglo XII la ciudad, que ya recibía anualmente en torno a 300.000 peregrinos, conoce su primer momento de esplendor, que se traduce en los grandes edificios eclesiásticos románicos, edificados por arquitectos y artífices foráneos llegados a través del Camino de Santiago. El estilo gótico se introduce en los siglos siguientes de manos de las órdenes mendicantes, que se asientan en enclaves extramuros, iniciando la expansión del perímetro urbano. En el XV, de la mano de los Reyes Católicos y posteriormente de la Universidad, establecida por Alonso III de Fonseca, se introduce un nuevo estilo; el renacimiento marca, por primera vez, una preeminencia de la arquitectura civil. A partir 
del XVI, con la reforma luterana, la peregrinación decae, pero entonces el culto jacobeo pasa a América, donde se fundan o se ponen bajo la advocación del Apóstol más de doscientas poblaciones. Pese al descenso de las peregrinaciones, las rentas eclesiásticas se mantienen gracias al voto de Santiago, que se recaudaba en toda España; esta pujanza económica hace posible la gran reforma barroca y neoclásica, a la que corresponde en gran medida la ciudad histórica que se nos ha legado: se derriba o, como mínimo, se encubre la arquitectura medieval para crear una nueva fisonomía de la escena urbana, acorde con el poder del señorío eclesiástico. Si en el XVI y la primera mitad del XVII son arquitectos foráneos (Gaspar de Arce, Mateo López, Ginés Martínez, Fernández Lechuga) los que planean esa reforma, desde mediados del XVII una generación de artistas gallegos (Domingo de Andrade, Simón Rodríguez, Casas Novoa, Sarela, Ferro Caaveiro, Lois Monteagudo) toma la alternativa y Compostela es, todavía, la ciudad más pujante de Galicia. En 1780 se publican las primeras Ordenanzas de Policía Urbana, que suponen una primera sistemática de la construcción y la urbanización. Pero en el XIX la actividad constructiva decaerá, pese a que caen las murallas y se elabora el primer ensanche de población. El cuadro político se torna adverso: en 1823 Compostela pierde la capitalidad provincial y pasa a depender de A Coruña. No obstante, hasta el mismo siglo XX siguen produciéndose reformas y sustituciones puntuales en el interior del recinto intramuros, mientras fuera de murallas se va desarrollando, de forma lenta pero decidida, la expansión urbana.

En 1944 se produce la declaración de conjunto histórico-artístico del centro histórico de Santiago y en 1947 se aprueba un nuevo plan de ensanche. En 1966 se aprueba un Plan General que, si bien establece zonas de protección en el perímetro del centro histórico, admite un exceso de volumen en las zonas edificables. Este Plan se revisará en 1974 con criterios desarrollistas, que propician un creciente proceso especulativo, favorecido por el crecimiento demográfico (debido, en buena medida, a la inmigración de las zonas rurales) y por las cuantiosas remesas de la emigración europea. La población estudiantil también crece exponencialmente entre los años 70 y 1989, cuando se aprueba la segregación de las Universidades de Vigo y A Coruña.

Entre tanto, la tradición de la peregrinación jacobea se mantuvo, en uno de sus característicos momentos recesivos, gracias a la Iglesia, a las investigaciones promovidas desde la Universidad -no sólo la compostelana, sino también en Francia, Italia, Alemania o los Países Bajos$\mathrm{y}$ a la actividad de las asociaciones y organizaciones internacionales relacionadas con el Camino. A partir de un determinado momento se 
incorporan gradualmente a esta preocupación los municipios y poblaciones del Camino, las Comunidades Autónomas y la Administración Central. Algunas claves serían:

- 1982: Año Jubilar Compostelano; el Papa visita por primera vez Santiago; el Consejo de Europa concede a la ciudad su Bandera de Honor.

- 1984: Recomendación 987 del Consejo de Europa sobre las rutas de peregrinación, alentando la cooperación internacional para preservar los itinerarios.

- 1985: Se celebra en Gante la exposición Santiago de Compostela. 1000 años de peregrinación europea; el Consejo de Europa concede a la ciudad el Premio Europa, y la UNESCO la incluye en la lista del patrimonio mundial. Se aprueba la Ley de Patrimonio Histórico Español.

- 1986: El MOPU encarga un Proyecto de actuación conjunta para la rehabilitación del Camino de Santiago.

- 1987: El Consejo de Europa proclama el Camino de Santiago como primer itinerario cultural europeo.

- 1991: Refundación del Real Patronato de la Ciudad de Santiago.

- 1993: Año Jubilar Compostelano; la UNESCO incluye el Camino de Santiago en la lista del patrimonio mundial.

Hasta el punto de inflexión que representan las elecciones municipales de 1979, Santiago de Compostela había vivido —como cualquiera de las viejas ciudades de España- de las rentas del pasado, sin plantearse ningún porvenir. El advenimiento de la democracia asigna a las ciudades nuevos roles. En nuestro caso, se trata de la capitalidad de la Comunidad Autónoma, aprobada en 1982, junto a eventos tradicionales como los años jubilares, que se celebran en ciclos regulares de $6-5-6-11$ años (cada vez que la festividad de Santiago, 25 de julio, coincide en domingo) o a episodios extraordinarios como la capitalidad europea de la cultura en el año 2000. La ciudad asume este papel a la par que se desarrolla y se dota de nuevo contenido su dimensión histórica. A partir de ese momento, la transformación promovida desde los sectores público y privado se lleva a cabo con una conciencia clara de que nada nuevo será duradero si no parte de una relectura crítica del patrimonio construido. Se emprende así la doble tarea de transformar y conservar en el lugar común de la ciudad histórica.

\section{Objetivos y estrategia del planeamiento urbano}

Para Compostela, principio y fin del Camino de Santiago, el último cuarto del siglo XX supone un vuelco y una actualización de sus funciones 
tradicionales. En términos políticos, como centro de decisión de Galicia, se constituye en referente de la Comunidad Autónoma y asume su histórica vocación internacional desde un concepto de Europa como suma de diferencias, con una nueva cultura y una conciencia emergente de ciudadanía. En el terreno económico, se desarrollan actividades vinculadas fundamentalmente al sector servicios: algunas ya consolidadas, como la secular dimensión universitaria y el comercio, otras que responden a una creciente demanda social - turismo, cultura - y las derivadas de la administración, que generan a su vez una demanda industrial cualificada de empresas de desarrollo tecnológico.

La reflexión sobre la esencia de la ciudad, en la línea antes definida de conservación y transformación, se manifiesta en una filosofía de la morfología urbana basada en dos polos complementarios: al oeste, la fachada de poniente se corresponde con el núcleo histórico desarrollado en torno a la catedral, con la idea mítica del ocaso, del finis terrae, que subyace en la leyenda jacobea. En este lugar se ha de actuar con un criterio de "reconstrucción crítica» y sin miedo a la modernidad, ordenando, conservando, rehabilitando, construyendo, peatonalizando, cuidando lo grande y lo pequeño. La fachada de levante, que históricamente tuvo un desarrollo menor, será el lugar del crecimiento mediante la implantación de las infraestructuras y equipamientos adecuados a los nuevos roles de la ciudad, evitando la excesiva presión inmobiliaria sobre la ciudad histórica.

A lo largo de quince años se ha trabajado entre el planeamiento y la arquitectura, a veces uno primero y otra después, pero generalmente de forma simultánea. En 1988, tras tres años de trabajo, se aprueba la revisión del Plan General de Ordenación Urbana, que amplía la delimitación de la ciudad histórica a $170 \mathrm{Ha}$. Su principal objetivo es lograr una urbe moderna, bien dotada de infraestructuras y equipamientos, socialmente equilibrada, con una habitabilidad bien distribuida y con un planeamiento y arquitectura de calidad. Este documento plantea la ordenación del territorio preservando las zonas montañosas y de cultivo, los cauces, el entorno monumental y los más de 100 pequeños núcleos de población comprendidos en el término municipal. De acuerdo con el criterio morfológico antes señalado, la fachada de poniente, con la ciudad histórica, el río y el monte, se protege física y ambientalmente; aquí se emplazan los dos campus universitarios exteriores al casco urbano. Sobre la fachada de levante se desarrollan de forma ordenada las infraestructuras y equipamientos, especializando la ciudad de norte a sur, a lo largo de un vial de 8 $\mathrm{km}$.: al norte, entre otros equipamientos, se desarrolla la zona industrial 
y se implanta un nuevo centro agropecuario y ferial; hacia el este, zonas deportivas, residenciales y de equipamientos (estadio de fútbol, palacio de congresos, centro comercial, etc.), y al sur otras áreas residenciales y de servicios especializados (comercial, hospitales). La autopista libre de peaje, con tres conexiones con el centro, desvía del núcleo urbano el tráfico de paso.

El proyecto urbanístico, así como el desarrollo de los distintos proyectos de edificación, cuenta con la participación de relevantes arquitectos nacionales e internacionales, cuya solvencia permite incorporar nuevas aportaciones que enriquecen el acervo patrimonial consolidado con elementos que constituyen un legado estrictamente contemporáneo. La doctrina de las intervenciones consiste en lograr una combinación entre la calidad arquitectónica que ayuda a cualificar el entorno -obteniendo un estado de opinión favorable, después de algunas polémicas iniciales-, y la práctica urbanística que introduce a través del planeamiento criterios de ordenación económica, social y de valoración cultural.

El Plan Especial del centro histórico, por su parte, se orienta a la conservación y restauración de la arquitectura, tanto monumental - religiosa y civil - como residencial, con la mejora de equipamientos y servicios, condición necesaria para mantener y potenciar su función central y su vocación institucional y económica. En el ámbito del plan convive una arquitectura tradicional de muy buena calidad con algunos espacios libres, que se ordenan para dotar a la ciudad histórica de nuevas zonas residenciales y terciarias. Un primer paso consistió en la elaboración de un censo, que arrojaba un saldo de 2.829 edificios, en los que se agrupan 6.484 viviendas (un 8,6\% de ellas vacías) y 1.883 locales comerciales (14,7\% desocupados); modificar este estado de cosas era uno de los objetivos del documento. El catálogo del patrimonio establece cuatro tipos de protección, clasificando 41 edificios monumentales de valor excepcional, 68 edificios singulares de alto valor, 293 edificios de especial interés tipológico y compositivo y 1.411 edificios de interés. Para todos ellos se establece una normativa detallada, manteniendo la estructura parcelaria, las características de la arquitectura exterior e interior y los materiales tradicionales - madera y piedratratados con nuevas tecnologías que permitan mejorar la habitabilidad. El catálogo incluye además las áreas de morfología destacada y define usos preferentes para la zona.

Pero el Plan Especial no se limita a la rehabilitación y la conservación, sino que, sin perder de vista el proyecto de ciudad al que antes se aludía, desarrolla operaciones encaminadas a recualificar el entorno, a corregir disfunciones y carencias y a sentar las bases para 


\section{Santiago de Compostela, conservación y transformación}

el adecuado desarrollo de ese proyecto. En este marco se inscriben determinadas realizaciones - complejos de Bonaval, el antiguo Burgo de las Naciones y el barrio de Vite, el tratamiento unitario de los tres campus universitarios, que se amplían con proyectos de Isozaki, Gallego y Siza - y actividades económicas y de ocio como la operación de San Lázaro-Amio. Todas ellas han tenido una repercusión considerable en sus entornos respectivos, pues en cada caso se ha estudiado cuidadosamente la implantación para alcanzar, más allá del proyecto concreto, un objetivo global: el papel recualificador de la arquitectura sobre el entorno, tanto en términos urbanos como económicos y sociales, y su efecto positivo sobre el medio, el papel curativo y restaurador de las erosiones producidas en el tejido histórico, y el propiamente funcional, como piezas que articulan y especializan las partes de la ciudad.

A título de ejemplo, pueden citarse algunas de estas realizaciones.

- Auditorio de Galicia. J. Cano Lasso, 1989.

Arquitectura y actividad cultural son en Compostela caras de una misma moneda. Para la primera intervención de envergadura en este proyecto de renovación urbana se eligió una vaguada en el margen de la ciudad histórica, creando un centro cultural dedicado a la música y las artes plásticas, en un edificio de línea sobria, hecho de piedra, cristal y cobre.

La ordenación del territorio circundante, donde predominan los usos universitarios, incluye amplias zonas verdes y un estanque. El vecino barrio de Vite - el primer polígono público de España, de los años 60 - se transforma con la dotación de nuevos equipamientos y la rehabilitación de las viviendas sociales, lo que se traduce en una sensible mejora de las condiciones de vida y en la consiguiente disminución de la problemática social.

- Centro Gallego de Arte Contemporáneo. A. Siza Vieira, 1993.

Intervención comprometida y bien resuelta en pleno tejido histórico, al lado de un edificio monumental, en un enclave de vocación cultural y educativa, con museos y centros escolares. El edificio completa una calle inacabada, dialogando con la arquitectura del entorno a través de un lenguaje deliberadamente austero, que consigue realzar el barroco del contiguo ex-convento de Bonaval. Los espacios interiores, de color blanco y madera, se distribuyen sobre una planta en $\mathrm{V}$, en tres niveles de salas, dependencias de trabajo y servicios complementarios. La arquitectura interior es de una sobriedad poco común, donde la modernidad se expresa sin ninguna concesión. 
En el exterior, englobando el conjunto, la huerta del convento y el antiguo cementerio municipal convertido en parque público, conservando la arquitectura funeraria - de tan singular significación en Galicia-, los muros de contención, los manantiales, las especies vegetales, e incorporando algún mobiliario puntual y esculturas de Chillida, Miyawaki y Nóvoa.

- Palacio de Congresos y Exposiciones. A. Noguerol y P. Díez, 1994.

San Lázaro es un barrio antiguo, aunque periférico, que flanquea un tramo del Camino Francés; su nombre indica claramente su antigua condición de lazareto y sugiere su situación relativamente marginal respecto del centro histórico. Este fue el lugar escogido para construir un nuevo equipamiento concebido para acoger la intensa actividad congresual de la ciudad.

Realizado en hormigón, vidrio y acero sobre una plataforma pétrea, se desarrolla en planta con dos amplios vestíbulos para exposiciones y una gran sala central para 2.200 espectadores, divisible mediante un telón hidráulico. Una segunda sala con 500 asientos y los servicios de restaurante y cafetería completan la distribución de la planta principal. En la superior se disponen las aulas y salas de seminarios para el desarrollo de las actividades propias de este tipo de eventos.

- Pabellón polideportivo de San Clemente. J.P. Kleihues, 1995

En un solar situado en la ciudad histórica, junto a un instituto de enseñanza media, se ha construido un nuevo equipamiento deportivo escolar. Previamente se produjo un fuerte debate sobre el uso del solar y del propio instituto: frente a una mayoría de la opinión que pretendía su conversión en Parlamento autonómico, el Ayuntamiento y la comunidad escolar sostuvieron la necesidad de mantener un equipamiento público educativo en la ciudad histórica, mejorando al mismo tiempo las dotaciones de la zona con un estacionamiento subterráneo y locales comerciales.

La polémica toma como pretexto la introducción materiales y modulaciones distintos a los tradicionales: un pabellón de piedra, vidrio y cobre se encaja entre dos edificios preexistentes, uno histórico y otro historicista, pero moderno, y su transparencia facilita la integración. En realidad, lo que se debatía era una cuestión de jerarquía, defendiendo el Ayuntamiento la precedencia del planeamiento vigente sobre los criterios de la Comisión de Patrimonio histórico-artístico.

- Colegio de enseñanza primaria. G. Grassi, 1995.

La implantación de arquitectura de calidad en los barrios demuestra una vez más, en esta operación, su capacidad para ordenar la periferia, 
Santiago de Compostela, conservación y transformación

generando nuevos usos y actividades de forma racional y mejorando la calidad de vida. Esta actuación está incluida en el anteproyecto del plan especial de ordenación y protección del cauce del río Sarela, que delimita el centro urbano por el oeste. La planificación de la zona incluye la construcción de un pabellón polideportivo anexo al colegio.

La arquitectura escolar, de ordinario poco cuidada en su diseño, recibe un tratamiento cuidadoso en este edificio. Su simplicidad de formas, unida a la calidad constructiva, cualifican el patrimonio edificado de un barrio periférico, en el que gradualmente ha ido asentándose un importante contingente de población.

- Remodelación de la Avda de Juan XXIII. Piñón y Viaplana, 1996

En los años 60 el asfalto se había introducido de forma abrupta en la ciudad histórica a través de la principal vía de entrada de turistas, rompiendo transversalmente el substrato territorial preexistente. Para restañar esa fractura, se remodela el vial y se crea bajo la rasante una edificación para la dotación de nuevos servicios, en tres niveles diferenciados: en el inferior una dársena para autobuses turísticos, en el intermedio la zona de recepción de público y un estacionamiento subterráneo, y en el superior un bulevar peatonal. La esbelta marquesina de acero y vidrio define un espacio de $300 \mathrm{~m}$. de longitud que conecta con la ciudad histórica, logrando un atractivo efecto fugado. En la cota más baja del terreno se construyó un pabellón polideportivo para el centro escolar próximo.

Aquí se reprodujo la polémica sobre la superposición de arquitecturas, que ha sido característica en Compostela en todos los tiempos: cada vez que se construye un nuevo edificio singular se manifiesta la división de opiniones. Este fue un caso típico: ¿podía alterarse la perspectiva con un objeto nuevo que irrumpe notoriamente? ¿con qué altura, con qué autoridad? La única respuesta es la que dimana del plan y del proyecto, que implican a su vez el factor tiempo, pues el tiempo permite ir modelando opiniones y pareceres que contribuyen a hacer bien las cosas y avalan, en cierta medida, la pertinencia de las intervenciones.

\section{La rehabilitación}

La rehabilitación no es tanto una cuestión de materiales o de subvenciones, cuanto una reflexión interdisciplinar sobre los problemas del centro histórico para darles respuesta adecuada. En 1994, el Consorcio de la Ciudad, organismo ejecutivo del Real Patronato de Santiago de Compostela, en el que están representadas las administraciones 
central, regional y local, encomienda al Ayuntamiento el establecimiento de las medidas oportunas para la rehabilitación de la ciudad histórica. Con la creación de una Oficina Técnica, el Consorcio inicia un ambicioso proyecto que armoniza la necesaria renovación de las viviendas con el respeto y la conservación de todos los elementos que identifican y caracterizan estas arquitecturas.

Mientras se procedía a la redacción y tramitación del Plan Especial, se puso en marcha un plan puente de ayudas a la rehabilitación. Su primer objetivo era crear o recuperar entre los propietarios y los residentes la cultura de la conservación y el mantenimiento de las viviendas y locales comerciales. En un primer momento se acometieron actuaciones en el exterior de los edificios: fachadas, cubiertas, bajantes, carpintería... Los sucesivos programas, abordando ya intervenciones en interiores, tuvieron una función eminentemente didáctica y persuasiva, convenciendo a los ciudadanos de los beneficios de la rehabilitación y de la necesidad de implicarse en la tarea junto a las administraciones públicas y a los técnicos. Éstos, a su vez, debían ser conscientes de que se trataba de intervenir en la biografía personal y colectiva depositada en los estratos de la vivienda y del entorno.

La Oficina Técnica se ha ocupado de organizar las estructuras operativas que intervienen en la rehabilitación, los técnicos y empresas que han hecho posible un proceso compartido y aceptado por los ciudadanos. El programa cuenta con empresas de rehabilitación homologadas, a las que se imparten regularmente cursos prácticos sobre técnicas y materiales, y que mejoran su servicio a través de la experiencia en las intervenciones programadas y permanentemente supervisadas por la Oficina técnica. Arquitectos de la ciudad colaboran en la dirección de las obras en períodos rotativos de seis meses y con dedicación total al programa, mientras que otros arquitectos completan su formación académica a través del Aula de Rehabilitación, participando en la redacción de proyectos y en el control de obras.

Todos los proyectos se realizan en la Oficina, excepto aquellos considerados como obra mayor o muy compleja, que son encargados por los propietarios a arquitectos de su elección. En la Oficina encuentran los particulares la asistencia técnica y administrativa que precisan para definir el alcance de las obras, sus presupuestos y la elección de la empresa que desarrollará los trabajos. También se les facilita asesoramiento arqueológico y la tramitación de permisos y licencias de obra. Desde el primer contacto, los técnicos de la Oficina se ocupan de cada ciudadano, visitando su vivienda para tratar de conciliar sus 
deseos y necesidades con las exigencias imprescindibles para asegurar la conservación del patrimonio.

A lo largo de siete años el programa de rehabilitación ha consolidado un sistema caracterizado por la agilidad de la tramitación administrativa, la asistencia técnica integral y la relación directa, personal y cualificada con cada uno de los agentes implicados, pues desde que el propietario recurre a este servicio, un solo órgano administrativo realiza toda la tramitación del proyecto, selección del contratista, asistencia técnica, etc. Y, sobre todo, un sistema basado en la economía de las intervenciones ligeras y en la flexibilidad de la rehabilitación dispersa, programada conforme a las directrices del Plan Especial de Protección y Rehabilitación de la ciudad histórica. La rehabilitación ligera se considera un instrumento óptimo para la conservación de las ciudades históricas, frente a las operaciones «macro» de renovación urbana dirigidas desde la administración. Además, la inversión por vivienda es asequible con o sin créditos de financiación, y colectivamente positiva.

Tras el desarrollo de los programas de vivienda, se puso en marcha una línea de intervención en locales comerciales y, posteriormente, se ha abordado la restauración y conservación de edificios singulares: iglesias, teatros, mercados... He aquí algunas cifras indicativas del trabajo desarrollado:

\begin{tabular}{|l|c|c|}
\cline { 2 - 3 } \multicolumn{1}{c|}{} & \multicolumn{1}{c|}{$\begin{array}{c}\text { Programa de vivienda } \\
\text { (noviembre 1994-agosto 1999) }\end{array}$} & $\begin{array}{c}\text { Progr. establec. comerciales } \\
\text { (enero 1996-agosto 1999) }\end{array}$ \\
\hline total de obra finalizada & 1.832 .318 .174 pta. $=11.012 .454$ euros & 169.192 .156 pta. $=1.016 .856$ euros \\
\hline $\begin{array}{l}\text { total obras no } \\
\text { subvencionadas }\end{array}$ & 1.212 .371 .275 pta. $=7.286 .498$ euros & 123.203 .319 pta. $=740.467$ euros \\
\hline total subvencionado & 619.946 .900 pta. $=3.725 .956$ euros & 45.988 .836 pta. $=276.398$ euros \\
\hline obras finalizadas & 538 & 51 \\
\hline importe medio & 3.405 .796 pta. $=20.468$ euros & 3.317 .493 pta. $=19.939$ euros \\
\hline subvención media & 1.152 .318 pta. $=6.925$ euros & 901.742 pta. $=5.420$ euros \\
\hline
\end{tabular}

En cuanto al número de licencias, en buena parte promovidas por la iniciativa privada, sin recurrir a las subvenciones tramitadas por la Oficina de Rehabilitación, en los dos años transcurridos desde la aprobación del Plan Especial, los datos son los siguientes:

- 90 edificios

- 323 viviendas

- 435 locales comerciales

- 8 hoteles 
El balance de la experiencia indica, además, que las nuevas tecnologías, coherentemente aplicadas, resuelven satisfactoriamente los problemas de la edificación histórica y la conservación de las estructuras tradicionales, dando respuesta a la demanda de habitabilidad y calidad ambiental sin modificar la esencia del patrimonio. Con las técnicas adecuadas, es posible la reparación y mantenimiento continuos de la vivienda, adaptándose a las necesidades que van apareciendo en la vida de los usuarios y sin necesidad de afrontar grandes costes.

\section{Movilidad y calidad ambiental}

Con las nuevas funciones administrativas y el creciente flujo turístico, el uso del vehículo privado empieza a generar tensiones en el centro. En el centro histórico se adoptan de inmediato medidas tendentes a preservar y potenciar los usos peatonales, regulando el acceso de los residentes y las operaciones de carga y descarga. La modernización del transporte público compromete tanto las líneas urbanas como las conexiones con el centro ciudad de los puntos de recepción de viajeros: el aeropuerto internacional (1,2 millones de pasajeros en 1999), la estación de ferrocarril (más de 2 millones) y la estación de autobuses (6 millones anuales). En cuanto al tráfico de paso, la entrada en servicio de la autovía periférica en 1994 complementa el servicio del tramo de autopista libre de peaje, aliviando la densidad del tráfico en el casco urbano y transformando la tradicional estructura radiocéntrica de transporte. Como ya se indicó, sobre este vial se han asentado los nuevos equipamientos públicos y privados - desarrollo del polígono industrial, mercado de ganados, palacio de congresos, hospitales, centros comerciales, instalaciones deportivas- que disponen de sus respectivos aparcamientos de gran capacidad.

El problema que representa el tráfico regional depende fundamentalmente de dos factores: la tradicional actividad comercial que opera sobre un amplio hinterland territorial, y el reciente desarrollo residencial de los municipios limítrofes, con una normativa urbanística más permisiva respecto a la conservación del patrimonio natural y del hábitat tradicional. El desplazamiento masivo de estudiantes en los fines de semana tiende a utilizar más bien el ferrocarril y el autobús. Para atender esta demanda, en el anillo que rodea la zona monumental, en espacios libres sin interés arqueológico, se han creado 2.300 plazas de estacionamiento, entre las que destaca por sus características funcionales la ya descrita de Juan XXIII. No obstante, se ha tenido presente que la construcción de estacionamientos en el centro es un factor que 


\section{Santiago de Compostela, conservación y transformación}

incrementa la atracción del tráfico rodado. Por ello se ha establecido un segundo anillo de estacionamientos céntricos, con 2.400 plazas, y se está desarrollando sobre las principales vías de acceso una red periférica que supera en número de plazas la suma de los dos anteriores, y que se comunicará con el centro mediante líneas de transporte público.

El control de tráfico permite mantener una buena calidad ambiental, con unas cotas bajas de contaminación atmosférica y acústica. Pero lo que ha contribuido de modo fundamental a este resultado ha sido el desarrollo de un cinturón verde en torno al centro histórico, con la ordenación de más de un millón de metros cuadrados de superficie. Así, a la centenaria Alameda, el jardín urbano por excelencia, se han ido agregando los parques Música en Compostela, Gijón, Bouza Brey, Pablo Iglesias, Almáciga, Bonaval, Belvís y Pajonal, comunicados de forma casi continua a través de corredores verdes.

\section{La gestión cultural}

La ciudad como proyecto cultural, un reto que se planteó desde el mismo principio como una respuesta o corolario inevitable de la actividad universitaria y turística, ha de considerar la producción y la reproducción de la cultura, lo que implica tener presente en todo momento la sociedad que ha de plasmar ese proyecto. Se trata de conseguir una mayor participación, no sólo en cuanto al público espectador sino, sobre todo, en un estímulo al proceso creativo que se refleje en una mayor proyección exterior.

Compostela había llegado a los años 80 con una penuria lamentable de infraestructuras culturales que pudiesen dar respuesta a una demanda muy cualificada. Así, en 1987 se recupera para la ciudad el Teatro Principal, que se convierte en el primer recinto público de producción cultural. En 1989 se inaugura el Auditorio de Galicia, el primero de la Comunidad Autónoma, que se constituye enseguida en referente ineludible mediante una programación ambiciosa y regular, esencialmente en los campos de la música y las artes plásticas; desde 1996 el Auditorio es la sede de la orquesta Real Filharmonía de Galicia y de la Escuela de Altos Estudios Musicales. En 1993 el Centro Galego de Arte Contemporánea, dependiente de la Xunta de Galicia, viene a abrir un nuevo frente en lo que se refiere a las diversas manifestaciones del arte de vanguardia. Gradualmente, a estos y otros recintos públicos menores va sumándose una red de espacios privados que hacen aportaciones diversificadas a la producción cultural. 
Junto a la programación ordinaria que durante la temporada promueven la iniciativa pública y privada, aprovechando los estímulos y recursos derivados de la actividad generada desde todos los sectores, se originan un conjunto de manifestacioneŝ extraordinarias que cuentan tanto con los grandes eventos de mayor repercusión como con los exponentes de la cultura internacional y de las denominadas como otras culturas. Es por ello que Compostela llega a ser designada como candidata a la capitalidad europea de la cultura para el año 2000. La Unión Europea le reconocerá ese título junto a Aviñón, Bergen, Bolonia, Bruselas, Cracovia, Helsinki, Praga y Reikiavik. Y precisamente en el umbral de ese año, la Xunta de Galicia acuerda construir una gran Ciudad de la Cultura en la falda del monte Gallás, según la propuesta ganadora del concurso convocado al efecto, firmada por Peter Eisenman.

\section{El turismo}

Se ha dicho que el turismo y la televisión han sido los dos grandes fenómenos sociales de la segunda mitad del siglo XX. En Compostela, como en todas partes, el fenómeno turístico experimentó un notable incremento a partir de los años 80, pero es 1993 el que marca el inicio de un «boom» que, en el horizonte de los años jubilares de 1999 y 2004, demandaba la creación y adaptación de sus infraestructuras públicas y privadas.

En el conjunto del patrimonio cultural, el legado arquitectónico es el que suscita mayor consenso en cuanto a su apreciación social, y este hecho es la base del llamado turismo «de calidad», como es el caso compostelano. Pero tras los primeros años de euforia se ha hecho evidente que este patrimonio no se renueva, sino que es un bien fungible, y su uso turístico indiscriminado constituye una amenaza potencial de la misma entidad que la contaminación ambiental. Las mediciones y controles realizados han confirmado, en efecto, el deterioro que la masificación turística provoca, incluso en los elementos aparentemente menos sensibles, como son los edificios.

Para afrontar este problema se ha diseñado una política de sostenibilidad, tratando de reconducir el fenómeno más a la «digestión» que a la «ingestión» del flujo turístico. La presión de éste se ejercía, fundamentalmente, mediante el tráfico de autocares y vehículos privados. Para evitar la degradación del centro histórico y mejorar las condiciones ambientales se adoptaron medidas como la construcción del área de recepción e información de Juan XXIII, en la que ya era la principal vía de entrada del turismo en el centro. 


\section{Santiago de Compostela, conservación y transformación}

La estructura hotelera, que había permanecido prácticamente inmutable entre 1982 y 1993, experimenta desde 1999 un notable impulso cualitativo y cuantitativo, no sólo en el número de plazas, sino en la categoría y estilo de los establecimientos. El sector sabe que, frecuentemente, cantidad es lo opuesto a calidad, y que en Compostela no debe prestarse atención sólo al turismo estacional, sino que debe tenerse presentes a los peregrinos que llegan a lo largo de todo el año, a un nutrido segmento de estudiantes que son, de hecho, transeúntes y a un tipo de visitantes muy cualificado, como es el turismo cultural, el de congresos y el de empresa, que tiene amplia presencia y desarrollo en la ciudad.

Pese a todas las consideraciones que se habían anticipado a la luz de la experiencia de 1993, en el año jubilar de 1999 el fenómeno turístico alcanzó, como era de prever, dimensiones problemáticas, lo cual ha puesto a las entidades implicadas -Ayuntamiento y Comunidad Autónoma, pero también a la Iglesia - ante la necesidad inexcusable de adoptar medidas ordenadoras, como las que hasta ahora han demostrado su efectividad en el caso del crecimiento urbano. El año 2000 , con la capitalidad cultural europea, resultó menos masivo, pero el próximo año jubilar será el 2004 y para entonces tendrán que haberse establecido nuevos planes de actuación al respecto.

\section{Qué hacer: el consenso}

Para llevar adelante una transformación culta de la ciudad fue necesario poner en juego importantes recursos, y para afrontar un reto así sólo cabía una fórmula: la del consenso. Esto, en Compostela, se fraguó y cuajó en la refundación del Real Patronato de la Ciudad, auspiciado por los Reyes de España. Esta institución se dotó de un organismo ejecutivo, el Consorcio de la Ciudad, que reúne bajo la presidencia del Alcalde a las tres administraciones públicas: Ayuntamiento, Xunta de Galicia y Estado. En términos económicos, el Estado asume el $60 \%$ de la financiación de los programas, la Comunidad Autónoma el 35\% y el Municipio el 5\%. En el seno del Consorcio se acuerdan los programas que han de desarrollar el plan trazado para la ciudad. Tanto los presupuestos como su aplicación deben ser aprobados por unanimidad, y es en este ejercicio obligado del entendimiento entre las administraciones, junto con la capacidad proyectual, donde reside el éxito de las operaciones abordadas en los últimos años.

Los frutos de esta labor técnica y política se han plasmado en una serie de reconocimientos internacionales, además de los ya men- 
cionados: Premio de la Real Fundación de Toledo, Premio Firenze, Premio Manuel de la Dehesa, Premio Gubbio y, en 1998, el Premio de Urbanismo que la Unión Europea concede cada cuatro años a una ciudad en reconocimiento por la calidad del trabajo realizado.

\section{Bibliografía}

1 (1977) Proyecto y ciudad histórica. I SIAC. Colexio Oficial de Arquitectos de Galicia, Santiago de Compostela.

2 Costa, P. y J. Morenas (1989) Santiago de Compostela 1850-1950. Colexio Oficial de Arquitectos de Galicia, Santiago de Compostela.

3 DAldA, J.L. y E. BARDAJí (1990) Santiago de Compostela: el Plan Especial. En La Ciudad como proyecto. UIMP-MOPU, Madrid.

4 Estévez, X. (1999) Santiago de Compostela: el evento cultural-religioso como estrategia de desarrollo urbano. En P. Maragall (ed.) Europa próxima. Europa, regiones y ciudades. Universitat de Barcelona-UPC, 135-141.

5 Estévez, X. (2001) Santiago de Compostela, Spain. En R. Pickard (ed.) Management of Historic Centres. London, Spon Press, 223-242.

6 Estévez, X. (2001) Santiago de Compostela. Planteamiento y gestión. En J. Maderuelo (ed.) Arte público: Naturaleza y Ciudad, Lanzarote, Fundación César MAnrique, $55-98$.

7 GARCÍA CORTIZo et alli, "El impacto de la capitalidad en la estructura espacio-funcional del centro histórico de Santiago de Compostela». X Congreso de Geografía. Zaragoza, 1987, vol. II, 561-569.

8 García Iglesias, J.M. (dir.) (1993) Santiago de Compostela. Xuntanza, A Coruña.

9 López Alsina, F. (1988) La ciudad de Santiago de Compostela en la alta edad media. Santiago de Compostela.

10 MARTí, C. (ed.) (1995) Santiago de Compostela: la ciudad histórica como presente. Consorcio de la Ciudad de Santiago de Compostela.

11 Oikos (1975) Notas sobre el desarrollo urbano de Santiago de Compostela en la década de los 60. Ciudad y territorio 1/2, 95-106. 